\title{
Momentum Resolved EELS of Plasmons at Al Grain Boundaries without a q-Slit Aperture
}

\author{
Eric R. Hoglund ${ }^{1}$, James M. Howe ${ }^{1}$
}

${ }^{1}$ Dept. of Materials Science \& Engineering, University of Virginia, Charlottesville, VA 22904, USA

Electron energy loss spectroscopy (EELS) in a transmission electron microscope (TEM) is a valuable tool for characterizing the fundamental chemical, photonic, and vibrational behavior of materials with high spatial resolution. When performing traditional EELS, one integrates all momenta $(\boldsymbol{q})$ entering the entrance aperture into a single energy loss spectrum (ELS). Consequently, the collected data lack information that the dispersive nature of the phenomena may provide. For example, one can extract information about the local electron density $(n)$ from the ELS due to a plasmon excitation, but one can additionally determine the extent of interaction between individual electrons in the plasma, as well as the Fermi energy by characterizing its dispersion, i.e., using its $\boldsymbol{q}$ dependence [1]. A TEM can be outfitted with a specially designed $\boldsymbol{q}$-slit aperture, which allows selection of specific directions in $\boldsymbol{q}$-space to enter the EELS, but such $\boldsymbol{q}$-slits are not a typical installation. Hence, we devised a technique to obtain the $\boldsymbol{q}$ dependence of plasmon excitations at grain boundaries (GBs) with high spatial resolution, using a FEI Titan TEM at $300 \mathrm{kV}$ with a Tridiem Model 863P Gatan imaging filter without a $\boldsymbol{q}$-slit.

To collect a $\boldsymbol{q}$-ELS, the specimen was then tilted into a systematic row condition corresponding to the direction in $\boldsymbol{q}$ space under investigation and a selected-area aperture (SA) was inserted around the area of interest. A $2 \mathrm{~mm}$ entrance aperture (EA) was used to minimize off-axis rays while maintaining the required region of $\boldsymbol{q}$-space under investigation. Acquiring an image of the ELS allowed $\boldsymbol{q}$-ELS patterns to be obtained, as shown in Figs. 1 and 2. Using this technique, the EA acts as a $\boldsymbol{q}$-slit, although it does not collect from a narrow slit, but rather from a circle. For this reason, it is important to control the quality of the diffracting condition by tilting so that only excitations of interest are collected. In Fig. 1a, between the zero-energy loss peak (ZLP) of the two Bragg reflections, two low intensity Bragg excitations are identifiable due an imperfect systematic row. Additionally, there is some curvature of the $q=0$ line introduced as an artifact of the spectrometer. These features show that the quality of the spectrum depends on the quality of the systematic row excitation and its position on the spectrometer, considerations that are not important when using a $\boldsymbol{q}$-slit. However, this technique can be performed on most EELS systems without the need for a special $\boldsymbol{q}$-slit aperture.

The microstructural features under investigation were GBs in a polycrystalline Al foil. Nandi et al. [2] showed that GBs have an electron density deficit using EELS, and that a interface plasmon resonance may be present. With this in mind, the above technique was used to further characterize the plasma behavior at a GB. A grain containing a [011] zone axis was located, and a SA was placed in the matrix away from the GB. $\boldsymbol{q}$-ELS were acquired for the (200) reflection and both variants of (111) reflections, as seen in Fig. 1. The diffuse intensity observed between the beams' ZLP, is due to phonon scattering. An energy loss due to excitation of a volume plasmon is evident at $15 \mathrm{eV}$, in addition to plural scattering events. The parabolic dispersive nature of the plasmons is apparent between the Bragg reflections and is identical regardless of the reflection. This demonstrates that the behavior of the electron gas is relatively isotropic in Al, in agreement with results given by Batson and Silcox [3]. The same procedure was performed so that the SA was adjacent to, but not containing, a GB. This condition ensured that reflections from the second grain did not contribute to the ELS. The resulting ELS for the (200) reflection and one of the (111) reflections are shown in Fig. 2. The (200) reflection contained scattering in a direction mostly perpendicular to the GB, where the (111) contained scattering mostly parallel to the 
GB. The $\boldsymbol{q}$-ELS in Fig. 2a for the (111) reflection appeared identical to the bulk $\boldsymbol{q}$-ELS in Fig. 1, with the dispersion extending 3-4 eV. In contrast, inspection of the scattering in the (200) direction perpendicular to the GB in Fig. 2b, showed the dispersion extended approximately $15 \mathrm{eV}$. It can therefore be concluded that the response of the electron gas is not significantly different from the bulk matrix along the GB, but the response is significantly altered perpendicular to the GB. These results demonstrate the utility of the proposed technique for collecting $\boldsymbol{q}$-EELS patterns and its potential to understand fundamental material properties.

\section{References:}

[1] R Friedrich et al, Journal of Electron Spectroscopy and Related Phenomena 195 (2014) p. 85-95.

[2] P Nandi et al, Microscopy and Microanalysis 23 (2017) p. 414-415.

[3] P Batson and J Silcox, Physical Review B 27 (1983) p. 5224-5239.

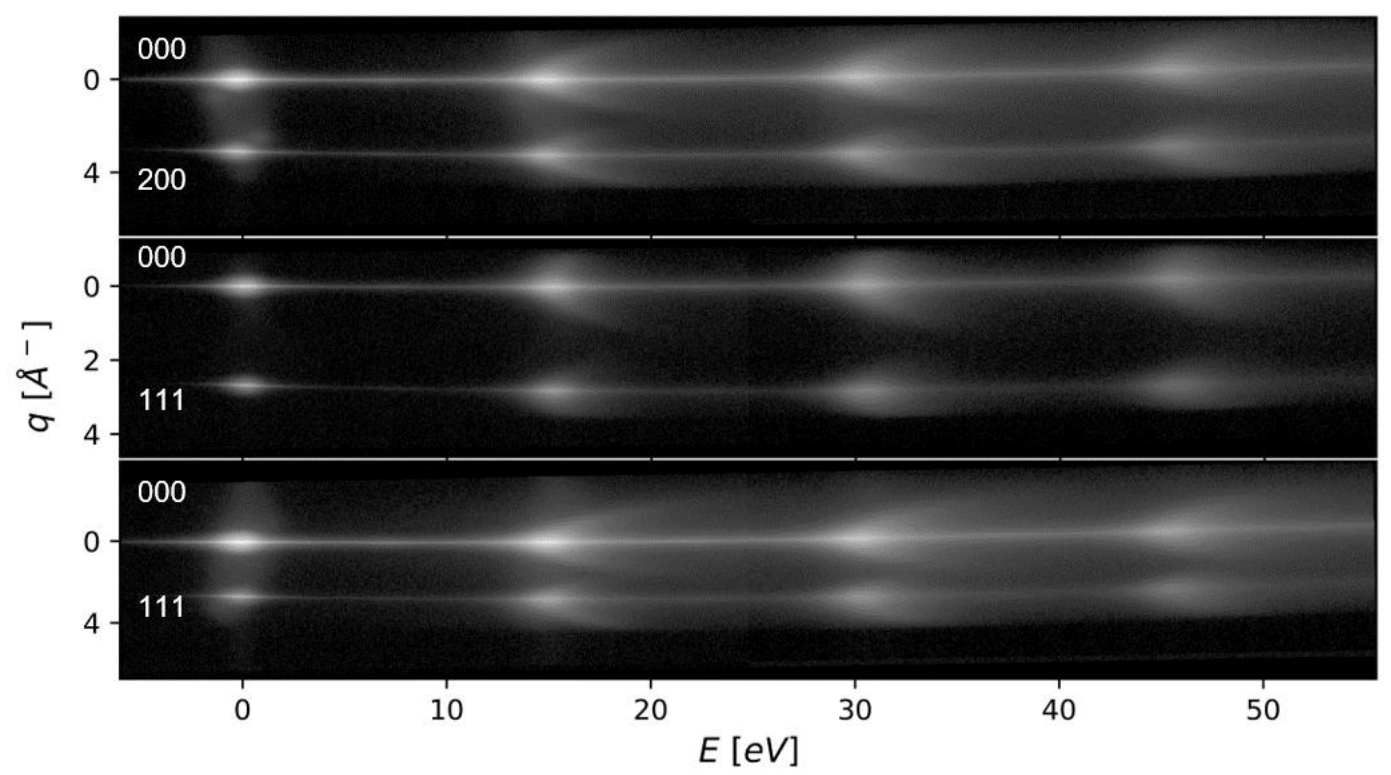

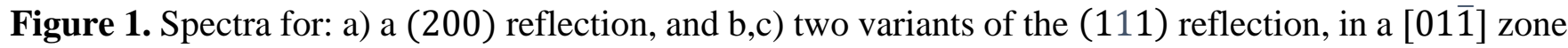
axis. All spectra were acquired in the interior of a grain. Plasmons are visible at $15 \mathrm{eV}$, and their dispersions appear relatively identical.

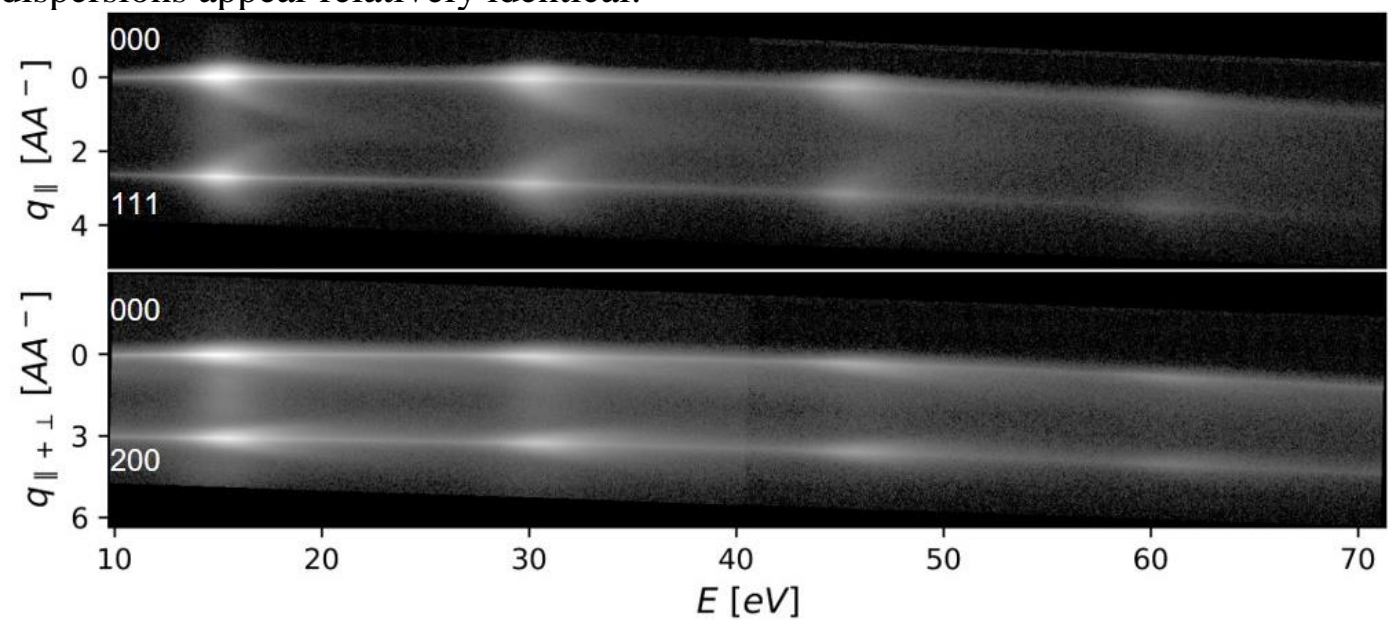

Figure 2. Spectra taken at a GB for a reflection: a) (111) mostly parallel to the GB, and b) (200) that contains a large component normal to the GB. The scattering behavior of the electron gas behaved identical to the matrix along the GB in a), but was modified perpendicular to the GB in $b$ ). 